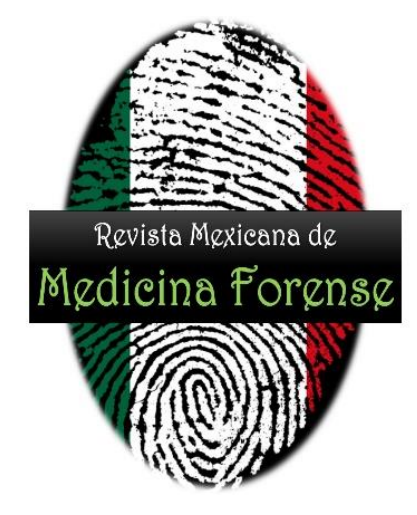

Rev Mex Med Forense, 2021, 6(1): 1-10

ISSN: 2448-8011

\title{
Características de descomposición y proceso entomológico en dos muestras de carne de cerdo Artículo Original
}

Decomposition characteristics and entomological process in two samples of pork

Granados-Muñoz, Rolando ${ }^{1}$; Robles-Hernández, Liliana ${ }^{2}$; González-Calette, Sergio J. ${ }^{2}$; Fajardo-Regalado, Verónica A. ${ }^{2}$

Recibido: 10 marzo 2020; aceptado: 7 Mayo 2020; Publicado: 15 Enero 2021.

${ }^{1}$ Licenciado en Criminología con Maestría en Ciencias del Comportamiento, profesorinvestigador de Criminología, Guanajuato, México. Director de la revista Archivos de Criminología, Seguridad Privada y Criminalística auspiciada por la Sociedad Mexicana de Criminología Capítulo Nuevo León.

${ }^{2}$ Esudiante Licenciatura en Criminalística, Grupo Educativo IMEI, Irapuato, Guadajuato. Corresponding author: Rolando Granados-Muñoz, r.granadosmunoz@ugto.mx. 


\section{RESUMEN}

El estudio de los insectos es de gran relevancia en áreas del conocimiento científico, su estudio suele darse en el ámbito forense y en estudios experimentales los cuales no son abundantes. Con el objetivo de describir las características de descomposición y el proceso entomológico que se presentan en dos muestras de carne de cerdo, se registraron estas muestras, una en ambiente controlado y la otra de manera expuesta, utilizando la técnica de observación se llevó a cabo el registro de medidas como volumen y peso, indicadores como coloración y deshidratación y el proceso entomológico. El experimento tuvo una duración de nueve días en el que se hicieron cinco observaciones, en los resultados obtenidos se identifican diferencias en la reducción de medidas y en la coloración y deshidratación, así como en la presencia de insectos entre ambas muestras. Esto permite establecer que, bajo ciertas condiciones, en este caso principalmente climáticas, se provocarán diferencias importantes en estos procesos.

Palabras clave: insectos, descomposición, entomología, muestra.

\section{SUMMARY}

The study of insects is of great relevance in areas of scientific knowledge; its study usually occurs in the forensic field and in experimental studies which are not abundant. With the purpose of describing decomposition characteristics and the entomological process that are presented in samples of pork, we registered one sample in a controlled environment and other in an exposed way, using the observation technique as well as measures such as volume and weight, indicators such as coloration and dehydration and the entomological process. The experiment lasted nine days; five observations were made; differences were identified in the reduction of measures, coloration and dehydration, as well as in the presence of insects between both samples. This allows to establish that, under certain conditions, in this case mainly climatic, important differences will be caused in these processes.

Keywords: insects, decomposition, entomology, sample.

\section{INTRODUCCIÓN}

El estudio de los insectos es de suma importancia en distintas áreas del conocimiento científico, no dejando de ser la excepción en el ámbito forense. La entomología forense es una de las disciplinas cuya aportación es de gran relevancia para el establecimiento post muerte de la persona privada de la vida (Vanegas \& Zamira, 2006).

Por otra parte, se encuentran los estudios entomológicos experimentales los cuales generalmente toman de referencia moscas y larvas para determinar diversas condiciones en los hechos relacionados con cadáveres. Suelen usarse muestras de carne de cerdo, se han hecho en ratas, conejos y hasta con un cadáver humano. Algunos de ellos toman como referencia las fases o etapas del proceso de descomposición.

Un ejemplo de estos estudios es el realizado por Armani, Dahinten y Centeno (2017) en el que buscaban determinar la composición específica, la abundancia y la diversidad de artrópodo fauna cadavérica asociada a un modelo experimental porcino. Realizaron muestreos estacionales durante tres años consecutivos. Observaron las cinco etapas 
del proceso de descomposición: fresco $(\mathrm{F})$, hinchado (H), descomposición activa (DAC), descomposición avanzada (DAZ) y restos (R). Recolectaron un total de 6325 individuos adultos e inmaduros, pertenecientes a 63 especies de 18 familias distribuidas entre los órdenes Diptera, Coleoptera e Hymenoptera, siendo los más importantes y numerosos desde el punto de vista de la entomología forense.

Otro estudio similar en moscas indica que la identificación de insectos encontrados en cadáveres es el primer paso que la entomología forense tiene en cuenta en la estimación del intervalo post mortem. Mediante el uso de trampas, es decir jaulas especiales, durante un período de siete meses, se utilizaron cerdos con un peso promedio de $18.75 \mathrm{~kg}$. De igual manera que en el experimento anterior, las fases de referencia fueron: fresca de 0-7 días, hinchada de 8-29 días, activa de 30-68 días, avanzada de 69-99 días y la de restos secos 99-190 días (Sánchez \& Fagua, 2014).

Por su parte Amat, Ramírez-Mora, Buenaventura y Gómez-Piflerez (2013) en su estudio con moscas carroñeras durante un año y dos muestreos mensuales, utilizando como cebo vísceras de pollo y cabeza de pescado, al recabar un total de 33834 moscas, establecen que, según la evidencia, la abundancia de moscas no varía según la época climática, pero sí respecto a las especies o la familia que llegaban.

En las investigaciones de entomología suelen analizarse también larvas, depositadas en cadáveres de cerdos domésticos, en sol y en sombra, para tomar muestras de estas y después de criarlas generar las descripciones de cada especie (Trigo, 2006). En México ha tenido relevancia, incluso se ha experimentado con el cuerpo humano para la recolección de larvas, específicamente para conocer su dispersión y áreas en que se presentan (Vergara-Pineda et al., 2012).

Incluso hay estudios que basan su análisis en la morfología de los insectos, Acuña-Morera, Cortés-Bernal, Vargas, Segura y Bello-García (2011) con el objetivo de identificar las características morfométricas cromosómicas y las estructuras primarias del cariotipo, a partir de especímenes de lucilia sericata, analizando los huevos embrionados, pudieron obtener características que permitieran la diferenciación de la especie. Algo de suma importancia pues bien postula Buenaventura (2012) admitiendo que las dificultades para establecer la identificación larval se deben al desconocimiento de su morfología y las características morfológicas de estas. A esto mismo hacen referencia Solano y Ramírez-Mora (2015) pues en su estudio destacan que en los casos de aplicación se debe realizar una correcta identificación de la taxonomía de las especies en algún estado de su desarrollo, acentuándose dicha dificultad en muestras deterioradas e incompletas.

Esto tiene soporte considerando la asociación de especies a los cadáveres y además de la relación con las fases de descomposición, en cadáver de cerdo o llama, en sol y en sombra (Aballay, Fernández, Mulieri \& Urquiza, 2011). Existe variación en el tipo de muestra, el tipo de insecto y las especies que se pretenden analizar o hasta el objetivo del estudio, en el realizado por Zhuang et al. (2011) hicieron uso de cadáveres de conejos, ellos formulan que las especies de insectos que se presentan tienen relación con la región en la que se realiza el estudio; en este mismo sentido Liria (2006) usó ratas de laboratorio con la finalidad de determinar la composición de fauna 
cadavérica y las especies que se presentan en las distintas etapas del proceso tafonómico.

Según algunas de las investigaciones de entomología que se han realizado, cada una de ellas mantiene su esencia en el propósito que persiguen, siendo coherente la asociación de los insectos con la descomposición cadavérica, misma que puede ser en muestras muy variadas. Con base en estas ideas se planteó un estudio con el objetivo de describir las características de descomposición y el proceso entomológico que se presentan en dos muestras de carne de cerdo, una en ambiente controlado y la otra de manera expuesta.

\section{MATERIALES Y MÉTODOS}

Se usaron dos muestras frescas de carne de cerdo en trozos de un kilogramo de peso cada una, se dividió en un ambiente control y otro expuesto, en la muestra expuesta se agregó un kilogramo de carne de cerdo entera al segundo día.

Muestra 1 (control). Esta muestra fue previamente medida y pesada antes de que se posicionará en el lugar, el cual estuvo localizado en el tercer piso de un edificio con puerta abierta y ventanas casi cerradas, con abertura no mayor a 5 centímetros, con la finalidad de que pudieran tener acceso a la carne los insectos. Debajo de la carne se le puso tierra para que mantuviera humedad. La principal condición de esta muestra es que estuviera en sombra, buscando la protección del sol y sus efectos sobre ésta, por ende, sobre los fenómenos entomológicos.
Muestra 2 (expuesta). En esta segunda muestra los pasos para montarla fueron similares, se hizo la medición y se tomó el peso (un kilogramo), se localizó en el mismo nivel (tercer piso), la exposición fue casi total a reserva de enjaular la muestra con el fin de evitar carroñeo de animales, los insectos podían tener acceso. Se le puso tierra por debajo. La principal condición fue que la muestra fuera afectada por los rayos solares y otros fenómenos climáticos. Se planteó adicionar un kilogramo extra entero de carne de cerdo si hubiese la presencia de insectos.

Para la evaluación de las muestras y el posterior análisis de los datos se utiliza la técnica de observación estructurada y no estructurada (Ibañez, 2014), en la primera se realiza de forma detallada y planificada con protocolos o instrumentos detallados, la segunda simplemente se observa anotando datos de manera subjetiva.

Se llevaron a cabo los registros en la Hoja de registro para la observación de la muestra (Anexo I) el cual hace referencia al llenado de datos de identificación y medidas de la muestra, se hicieron notas de campo en el lugar y durante el análisis de las fotografías tomadas, además se empleó la lista de cotejo para monitorear el proceso entomológico. En la manipulación y montaje de las muestras los utensilios fueron la cinta métrica, charola de aluminio, jaula, caja, báscula, cúter, papel plástico, guantes de látex, bata y cámara fotográfica.

\section{Procedimiento}

Lo primero que se hizo fue pesar la carne en trozos que tuvieran la medida de un kilogramo cada una, posteriormente ya teniendo los materiales para llevar a cabo 
los registros y las evaluaciones lo que se hizo fue poner la muestra 1 en sombra y poner la muestra 2 en un lugar expuesto que le diera la luz solar.

Las evaluaciones realizadas mantuvieron una duración de 30 a 50 minutos tres de cada cinco días según la duración del proceso entomológico, los registros fueron realizados por dos estudiantes en cada muestra. Cuatro estudiantes acudían el día lunes, otros cuatro el día miércoles y otros cuatro el día viernes.

Dentro de las actividades se preparaba el equipo para llevar a cabo la observación (bata, guantes de látex, cinta métrica, báscula, cámara fotográfica) y su posterior limpieza al termino de realizar la evaluación. La temperatura ambiente se realizó a través del teléfono celular, para el volumen de la muestra se marcaron cuatro puntos en cruz de referencia para cada medición, al final lo que se generó fue un promedio. En el caso del peso fue tomado considerando también el peso de la tierra. Una vez recabada la información lo que se hizo fue analizar los registros tomados en cada muestra.

\section{RESULTADOS}

Los registros fueron realizados en una hora promedio a las 09:00 horas y una duración promedio de 40 minutos. El experimento tuvo una duración de nueve días en el que se hicieron cinco observaciones, un total de 161 fotografías con presencia de dos días de lluvia. En la Tabla 1 se muestran datos de registro de la muestra 1 , con una temperatura promedio de $9.8^{\circ} \mathrm{C}$, un total de 80 fotografías y un volumen promedio de 27.2 centímetros.

\begin{tabular}{|c|c|c|c|c|c|}
\hline \multicolumn{6}{|c|}{$\begin{array}{l}\text { Tabla } 1 \\
\text { Registros de la muestra } 1 \text { por día de observación }\end{array}$} \\
\hline Día de observación & Uno & Dos & Tres & Cuatro & Cinco \\
\hline Temperatura ambiente $\left({ }^{\circ} \mathbf{C}\right)$ & 8 & 10 & 7 & 11 & 13 \\
\hline Número de fotografías & 18 & 17 & 20 & 12 & 13 \\
\hline Volumen (cm) & 28.5 & 27.5 & 27 & 26.5 & 26.5 \\
\hline
\end{tabular}

En la muestra 2 como se aprecia en la Tabla 2 la temperatura promedio fue de $9.6{ }^{\circ} \mathrm{C}$, un total de 81 fotografías tomadas y 25.7 centímetros de volumen promedio que, al igual que en la muestra anterior se distingue la reducción en cada día de observación, a excepción del día dos que aumenta por la adición del trozo entero de carne. Para el día nueve en la muestra 2 la carne estaba comida en su mayoría. 


\section{Tabla 2}

Registros de la muestra 2 por día de observación

\begin{tabular}{|llllll|}
\hline Día de observación & Uno & Dos & Tres & Cuatro & Cinco \\
\hline Temperatura ambiente $\left({ }^{\circ} \mathbf{C}\right)$ & 8 & 10 & 8 & 11 & 10 \\
\hline Número de fotografías & 7 & 19 & 24 & 19 & 12 \\
\hline Volumen (cm) & 26 & 28.5 & 26 & 25.5 & 22.5 \\
\hline Nota: ${ }^{\circ}$ C=grados centígrados, cm=centímetros. & \\
\hline
\end{tabular}

En lo que se refiere al peso, se presenta en la Figura 1 el registro de los pesos en kilogramos en cada uno de los cinco de días de observación, donde se identifican bajas de gramos excepto el día dos en la muestra expuesta porque se le adicionó el trozo de carne enteró.

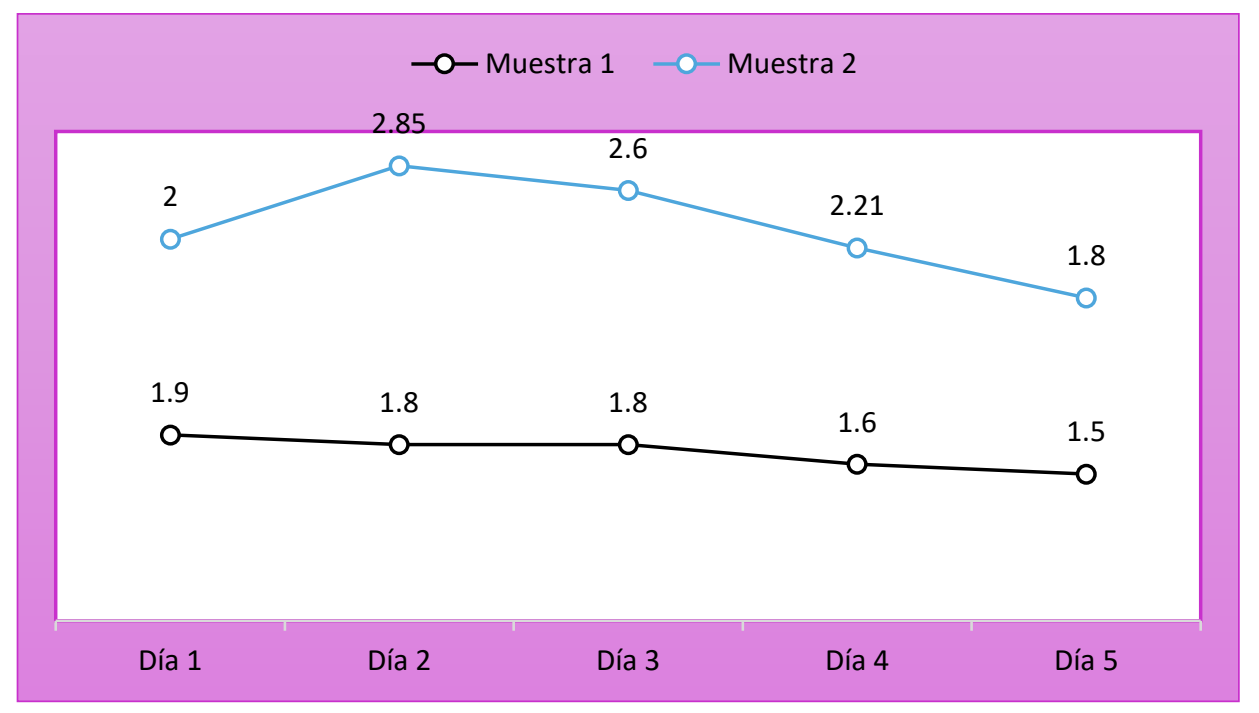

Figura 1. Registro de peso de ambas muestras por día de observación 
Indicadores de coloración y deshidratación

En lo que se refiere al indicador de color en la carne control (muestra 1) el proceso de pasar de un color rojo y rosa a una tonalidad morada y negra fue más lento respecto a la carne expuesta (muestra 2), la resequedad se fue dando marcadamente en esta muestra expuesta, incluso en la carne control hubo la presencia de hongo, según los registros estos fueron vistos a partir de la observación cuatro (día 7).

Muestra 1. Rosada y blanca, la carne estaba fresca (observación 1); rosa en el inferior o centros, roja y blanca, comienza a mostrarse dura (observación 2); morado en los extremos, rojo en el inferior y beige, la carne está dura en la superficie y blanda abajo (observación 3); negro-morado en los extremos, rojo en el inferior y beige, presenta una consistencia más dura y obscura (observación 4); negro-morado en los extremos con beige, la carne aun presenta humedad en el interior (observación 5).

Muestra 2. Rojo vivo y no hay deshidratación (observación 1); rojo vino y rojo con tonalidad más suave, deshidratación en la parte superior (observación 2); rojo vino en la parte superior y rosa en la inferior con presencia de humedad (observación 3); negra-rojiza con bastante resequedad (observación 4); rojo con negro y seca en su mayoría comida los trozos de carne (observación 5).

Algo importante referido en las notas es el olor que se desprendía, la muestra control tenía un olor penetrante, pudiendo ser por las condiciones del lugar cerrado, pero la carne mantenía aun cuando fue movida mantenía un aroma a descomposición diferente al de la muestra expuesta.

\section{Proceso entomológico en las muestras}

Como se verá en la tabla 3 , pese a que los insectos podían tener acceso en ambas muestras, a la muestra control llegaron hasta el día siete correspondiente al día cuatro de observación, y esto sucedió porque a partir de esta fecha el experimento se alteró y la carne fue movida a un lugar más abierto. Esto se dio de manera repentina y no fue un proceso paulatino como el que se presentó en la carne expuesta (muestra 2), hubo un retraso en la aparición de insectos y el desarrollo de estos ya no fue igual porque la carne ya no estaba fresca. 


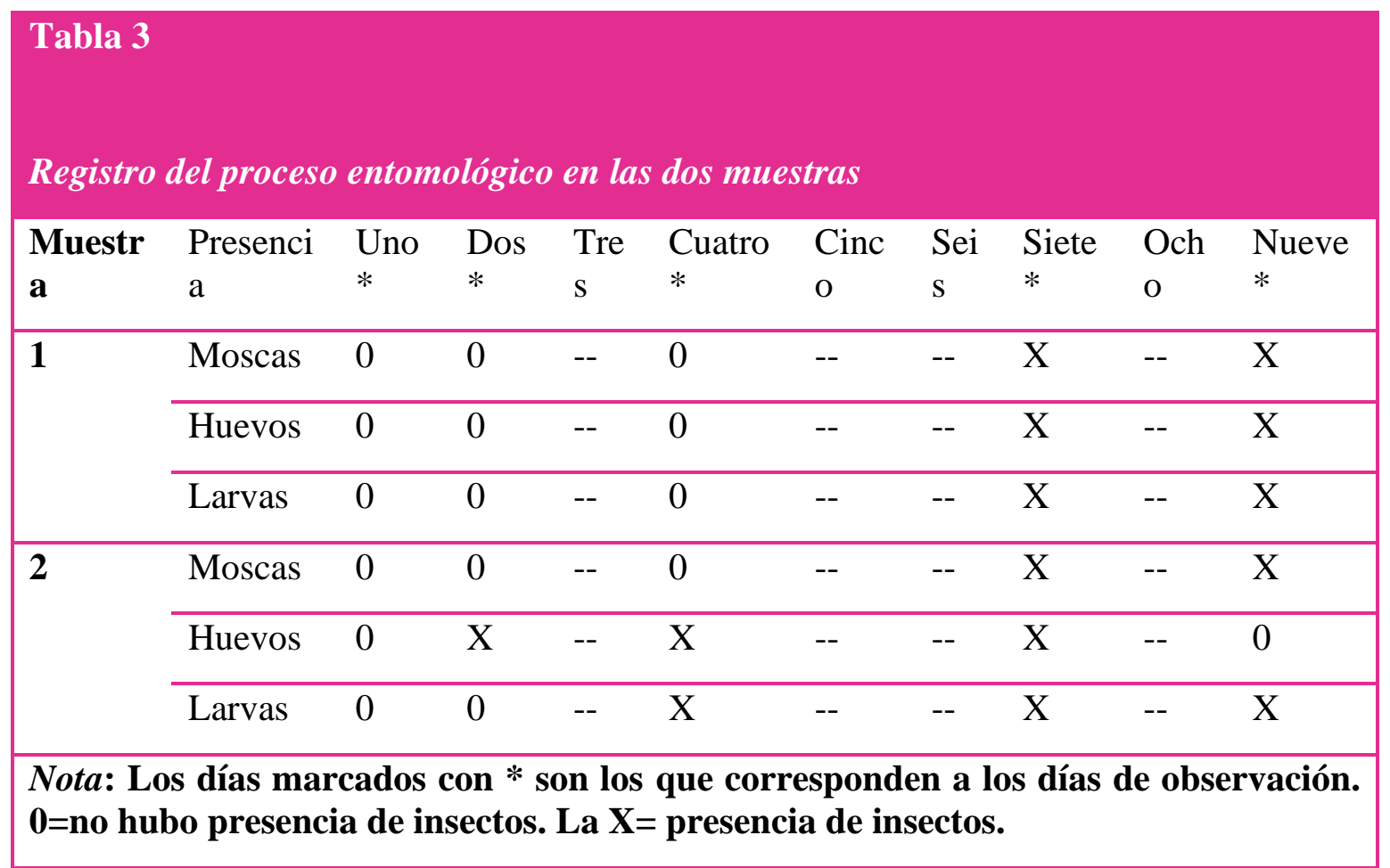

Algunos datos de importancia registrados en las observaciones hacen notar la manera de alimentación de las larvas, pues los huevos estaban depositados en las partes superiores y lugares húmedos, pero las larvas buscaban la carne fresca, es decir de las partes inferiores, comenzando por consumir la carne blanda.

\section{DISCUSIÓN}

En este estudio el propósito fue describir las características de descomposición y proceso entomológico de las muestras de carne de cerdo, con apoyo de los registros llevados a cabo durante nueve días se obtuvieron resultados aceptables que permiten establecer el cumplimiento de lo propuesto.
Desde el inicio se tenía previsto algunas limitaciones, por ejemplo, comparado con la temporalidad de estudios que duran meses y años (Amat, Ramírez-Mora, Buenaventura \& GómezPiflerez, 2013; Armani, Dahinten \& Centeno, 2017; Sánchez \& Fagua, 2014) a este experimento de nueve días, realmente no se pueden extender análisis más profundos, de ahí el objetivo demarcado en hacer las descripciones en cada muestra, sin embargo, existe la posibilidad de replicar estos mismos procedimientos, una de las intenciones de esta propuesta de investigación es que a futuro puedan realizarse metodologías similares teniendo presente la carencia de trabajos de este tipo.

Los resultados de las características de descomposición indican reducción de las muestras según el volumen y el peso, aunque no pueden ser monitoreadas las fases de descomposición (Armani, Dahinten \& Centeno, 2017; 
Sánchez \& Fagua, 2014), en las observaciones se constató que en la carne expuesta los cambios fueron más variables e impredecibles, porque en la carne control se registró consistencia, no sólo en las medidas sino hasta en la coloración y deshidratación, pues en esta muestra control fue una tonalidad de color obscuro incluso por la presencia del hongo, ha destacar en este caso fue la presencia de lluvia, que únicamente se presentó en la muestra expuesta. Esto da sentido a lo encontrado por Amat, Ramírez-Mora, Buenaventura y Gómez-Piflerez (2013), pues en el caso de los insectos el clima no es una condición para que aparezcan.

En el proceso entomológico no se pretendía como en otras investigaciones saber la composición de fauna cadavérica, cuantificar las especies o familias y su identificación que es de suma importancia para el intervalo post mortem, siendo la esencia de los estudios de entomología forense al analizar la morfología y el desarrollo de los insectos (Acuña-Morera, Cortés-Bernal, Vargas, Segura \& BelloGarcía, 2011; Amat, Ramírez-Mora, Buenaventura \& Gómez-Piflerez, 2013; Armani, Dahinten \& Centeno, 2017; Buenaventura, 2012; Liria, 2006; Solano \& Ramírez-Mora, 2015), la intención fue conocer el proceso entomológico al tener consciente el tamaño reducido de la muestra, de esta manera se conoció que la presencia de moscas, huevos y larvas fue más abundante en la muestra expuesta, incluso comprender lo que señalan Vergara-Pineda et al. (2012), acerca de las larvas ellos obtienen que la dispersión se realiza en los alrededores de la cabeza, según lo observado en este experimento al buscar carne húmeda y blanda, la cabeza resulta ser un lugar de fácil acceso por los orificios que tiene el cuerpo humano, entre ellos boca, nariz y orejas, aunado a la alimentación que representan los órganos de esta parte superior.

Las implicaciones de este estudio como de las demás investigaciones que se han realizado permiten generar una metodología de análisis que puede ser retomada y mejorada en trabajos que como ya se hizo mención no son abundantes. En especial al describir las características de descomposición y el proceso entomológico en las muestras de carne de cerdo empleadas permiten establecer que, bajo ciertas condiciones, en este caso principalmente climáticas, provocarán diferencias importantes en estos procesos.

\section{REFERENCIAS}

1. Aballay, F. H., FernándezCampón, F., Mulieri, P. R., \& Urquiza, S. V. (2011). Sarcophagidae (díptera) de importancia forense en la puna de Catamarca, Argentina: la ovoviviparidad como ventaja en condiciones de extrema aridez. Revista de la Sociedad Entomológica Argentina, 70(3-4), 255-266.

2. Acuña-Morera, Y., Cortés-Bernal, D., Vargas, M., Segura, N. A., \& Bello-García, F. (2011). Caracterización citogenética de lucilia sericata (Meigen, 1826) (diptera: calliphoridae), CEPA Bogotá, Colombia. Revista Ciencias de la Salud, 9(2), 111124.

3. Amat, E., Ramírez-Mora, M. A., Buenaventura, E., \& GómezPiflerez, L. M. (2013). Variación temporal de la abundancia en familias de moscas carroñeras (díptera, calyptratae) en un valle andino antropizado de Colombia. 
Acta Zoológica Mexicana, 29(3), 463-472.

4. Armani, A. P., Dahinten, S., \& Centeno, N. (2017). Artropodofauna cadavérica asociada a cerdo doméstico (sus scrofa) en un ambiente ribereño en Chubut, Argentina. Revista Colombiana de Entomología, 43(2), 262-267. doi: 10.25100/socolen.v43i2.5955

5. Buenaventura, E. (2012). Morphology of the first and second instars larvae of peckia (peckia) chrysostoma (Wiedemann, 1830) (diptera, sarcophagidae). Acta Zoológica Mexicana, 29(1), 96104.

6. Ibañez, J. (2014). Métodos, técnicas e instrumentos de la investigación criminológica. España: Dykinson.

7. Liria, J. (2006). Insectos de importancia forense en cadáveres de ratas Carabobo, Venezuela. Revista Peruana de Medicina Experimental y Salud Pública, 23(1), 33-38.

8. Sánchez, A. F., \& Fagua, G. (2014). Análisis sucesional de calliphoridae (diptera) en cerdo doméstico en pastizales (Cogua, Cundinamarca, Colombia). Revista Colombiana de Entomología, 40(2), 190-197.

9. Solano, K. V., \& Ramírez-Mora, M. A. (2015). Morfología de los genitales masculinos de tres especies de muscidae (insecta: diptera) de importancia forense en Colombia. Boletín Científico Centro de Museos Museo de Historia Natural, 19(1), 235-244. doi: $10.17151 / \mathrm{bccm} .2015 .19 .1 .17$

10. Trigo, V. (2006). Descripción de las larvas II, III y el pupario de compsomyiops fulvicrura (díptera: calliphoridae). Revista de la Sociedad Entomológica Argentina, 65(1-2), 87-99.

11. Vanegas, Y., \& Zamira, S. (2006). Entomología forense: los insectos en la escena del crimen. Revista Luna Azul, (23), 42-49.

12. Vergara-Pineda, S., LeónMúzquiz, H., García-Martínez, O., Cantú-Sifuentes, M., LanderosFlores, J., \& Tomberlin, J. K. (2012). Dispersión espacial de larvas de lucilia sericata y calliphora coloradensis (díptera: calliphoridae) en etapa de postalimentación. Revista Colombiana de Entomología, 38(1), 97-99.

13. Zhuang, Q., Cai, J., Zhang, M., Feng, H., Guo, Y., Lan, L., \& Chen, Y. (2011). Molecular identification of forensically significant beetles (coleoptera) in China based on COI gene. Revista Colombiana de Entomología, 37(1), 95-102.

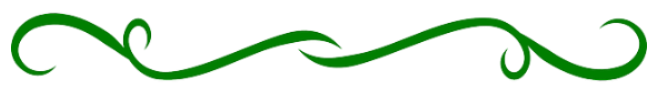

Revista Mexicana de Medicina Forense y Ciencias de la Salud 